\title{
Endothelin-1 Mediates Hypoxia-Induced Increases in Vascular Collagen in the Newborn Mouse Lung
}

\author{
NAMASIVAYAM AMBALAVANAN, PENG LI, ARLENE BULGER, JOANNE MURPHY-ULLRICH, SUZANNE OPARIL, \\ AND YIU-FAI CHEN \\ Department of Pediatrics [N.A., A.B.], Department of Medicine [P.L., S.O., Y.-F.C.], Department of Pathology [J.M.-U.], University of \\ Alabama at Birmingham, Birmingham, Alabama 35233
}

\begin{abstract}
Endothelin-1 (ET-1) mediates hypoxia-mediated pulmonary vascular remodeling (HPVR), and endothelin-A receptor (ET-AR) blockade prevents HPVR in newborn mice. Our objective was to determine postnatal effects of chronic hypoxia and/or ET-AR blockade on lung ET-1, ET-AR, ET-BR, and vascular collagen and elastin. Newborn C57BL/6 mice ( $n=6-8 / \mathrm{gp}$ ) given either BQ610 (ET-AR blocker) or vehicle were exposed to air or hypoxia $\left(12 \% \mathrm{O}_{2}\right)$ from birth for 1, 3, or $14 \mathrm{~d}$. Lung ET-1 was assessed by ELISA, and ET-AR and ET-BR by immunohistochemistry. Vascular collagen and elastin were assessed by quantitative image analysis. ET-1, ET-AR, ET-BR, collagen I and III, and tropoelastin mRNA levels were assessed by real-time quantitative RT-PCR. We observed that: 1) hypoxia attenuated the normal postnatal decrease in ET-1 and collagen content; 2) ET-AR blockade reduced collagen independent of $\mathrm{O}_{2}$;3) hypoxia increased elastin mRNA expression and attenuated the normal postnatal decrease in elastin content; and 4) BQ610 reduced elastin mRNA but not elastin content. We conclude that, in neonatal mice, hypoxia attenuates normal postnatal decreases in ET-1, vascular collagen, and elastin. ET-AR blockade reduces collagen fiber area but not mRNA, and does not decrease elastin despite reducing its expression. (Pediatr Res 61: 559-564, 2007)
\end{abstract}

A bnormal pulmonary vascular remodeling due to hypoxia (1) may lead to persistent pulmonary hypertension of the newborn (PPHN), which affects more than 10,000 infants annually in the United States (2). Pulmonary vascular remodeling also occurs in bronchopulmonary dysplasia (3) and congenital heart disease (4). Pulmonary vascular remodeling is more severe in neonates compared with adult animals following similar hypoxic exposure (5), and various neonatal animal models (5-7), including our recently developed newborn mouse model (8), have been developed for the evaluation of HPVR. Neonatal HPVR differs significantly from adult HPVR with respect to extracellular matrix composition, pulmonary arterial smooth muscle structure, and vascular reactivity (9).

Received August 28, 2006; accepted December 7, 2006.

Correspondence: Namasivayam Ambalavanan, M.D., 525 New Hillman Bldg., University of Alabama at Birmingham, 619 South 20th St., Birmingham, AL 35294; e-mail: ambal@uab.edu

Funded in part by the National Institute of Child Health and Human Development K08 HD046513, a grant from the CCRI, a Research Facilities Improvement Program Grant C06 RR 15490, ATS PH-06-006, and National Heart, Lung, and Blood Institute grants HL-44195, HL-50147, HL-45990, HL-07457, and HL-56046.

DOI: 10.1203/pdr.0b013e318045beae
HPVR is mediated in part by ET-1, a 21-amino acid polypeptide with two major receptor isoforms, ET-AR and ET-BR. ET-AR, located mainly on pulmonary arterial smooth muscle cells, mediate vasoconstriction, whereas ET-BR, located mainly on pulmonary endothelial cells, mediate vasodilation (10). $\mathrm{A}$ rare $\mathrm{ET}_{\mathrm{B}}$ receptor subtype $\left(\mathrm{ET}_{\mathrm{B} 2}\right)$, present on vascular smooth muscle cells, also mediates vasoconstriction (11). The biologic actions of ET-1 vary with age (12), vascular bed, dosage (13), and species (14). Hypoxic exposure leads to an increase in ET-1 release by pulmonary endothelial cells that acts by a paracrine mechanism on adjacent vascular smooth muscle cells to cause vasoconstriction and pulmonary hypertension, which, if sufficiently prolonged, leads to HPVR (15). Nonselective (combined ET-AR and ET-BR) antagonists have the disadvantage of inhibiting ET-BR mediated vasodilation, and selective ET-BR blockade worsens pulmonary hypertension by vasoconstriction via ET-AR without concomitant ET-BR induced vasodilation (15). We have shown that ET-AR blockade attenuates acute hypoxia-induced pulmonary hypertension in piglets (16), and that ET-AR antagonists attenuate and reverse HPVR in adult rats (17) and in newborn mice (8). However, while is known that ET-AR blockade can reduce hypoxic vasoconstriction and HPVR, the effects of ET-AR blockade on hypoxia-induced changes in ECM molecules has to be determined. In addition, the lung undergoes marked developmental changes in the first two postnatal weeks in the newborn mouse, and data on ET-1 and its receptors during this dynamic process would be valuable to better understand the role of ET-1 in normal and pathologic lung development.

The objectives of this study were to a) determine the normal postnatal changes and effects of chronic hypoxia on lung ET-1 and its receptors, and b) determine the effects of chronic hypoxia on collagen and elastin in resistance pulmonary arteries and to test whether ET-AR blockade prevents the hypoxia-induced effects. It was hypothesized that ET-1, ET-AR, ET-BR, vascular collagen, and elastin would be highest around birth and decline postnatally, that hypoxia would attenuate the normal postnatal decrease in these molecules,

Abbreviations: ECM, extracellular matrix; ET-AR, endothelin-A receptor; ET-BR, endothelin-B receptor; ET-1, endothelin-1; HPVR, hypoxia-induced pulmonary vascular remodeling 
and that ET-AR blockade would permit the normal changes in vascular collagen and elastin to occur.

\section{METHODS}

Experimental animals and animal care. The research protocol was approved by the Institutional Animal Care and Use Committee of the University of Alabama at Birmingham, and the care and handling of the animals were in accordance with National Institutes of Health guidelines. Timed pregnant C57BL/6 mice were obtained from Charles River Laboratories (Wilmington, MA).

Animal model. Newborn C57BL/6 mouse pups and their dams were exposed to normobaric hypoxia $\left(12 \% \mathrm{O}_{2}\right)$ or normoxia (room air) from birth to either postnatal d 1 or 14 ( $n=6-8 / \mathrm{gp}$ from at least two litters), while being given BQ610 (ET-AR antagonist) or vehicle (cottonseed oil) as described previously (8).

Mortality was comparable (10-20\%) to that in nonexperimental conditions, and was minimized by using experienced dams, reduced handling, extra nesting material, and a high fat diet. Surviving hypoxia exposed pups were active but were smaller than air controls. Hypoxic pups weighed approximately $4-6 \mathrm{~g}$, while normoxic pups weighed $6-8 \mathrm{~g}$ at $14 \mathrm{~d}$. Administration of BQ610 did not prevent the hypoxia-induced growth retardation.

After completion of the period of hypoxia/air exposure, the animals were euthanatized. For histologic analysis, the lungs were fixed in inflation using $10 \%$ formalin and the right ventricle was perfused with formalin. Histologic sections $(5 \mu \mathrm{m})$ consisted of a coronal section from apex to base of both lungs. For RNA isolation, lungs were homogenized in $1 \mathrm{~mL}$ of TRIZOL total RNA isolation reagent (Invitrogen, Carlsbad, CA). For protein analysis, lungs were homogenized in $1 \mathrm{~mL}$ of a tissue protein extraction reagent (T-PER, Pierce Biotechnology Inc, Rockford, IL), centrifuged at $7000 \mathrm{~g}$ for $5 \mathrm{~min}$, and the supernatant frozen at $-80^{\circ} \mathrm{C}$ until analysis.

ET-AR antagonist administration. BQ610 (Peptides International, Inc, Louisville, KY) or vehicle was given intraperitoneally at $20 \mu \mathrm{g} / \mathrm{g}$ body weight per day, as described previously (8). BQ610 at this dose is a selective $\mathrm{ET}_{\mathrm{A}}$ antagonist $\left(\mathrm{IC}_{50}\right.$ for $\mathrm{ET}_{\mathrm{A}}=0.64 \mathrm{nM}$, for $\left.\mathrm{ET}_{\mathrm{B}}=24 \mu \mathrm{M}\right)$, and was effective in our previous study (8).

Quantitation of ET-1. Homogenized lungs were analyzed as a single batch for ET-1 by ELISA (R \& D Systems, Minneapolis, MN) as described in the manufacturer's protocol. The range of measurement was $1-120 \mathrm{pg} / \mathrm{mL}$, with good intra- and interassay precision.

Immunohistochemical staining for ET-AR and ET-BR. Antigen retrieval was performed by heating slides in citrate buffer (Lab Vision Corp., Fremont, $\mathrm{CA}$ ) for $20 \mathrm{~min}$. Slides were then stained with rabbit polyclonal antibodies to ET-AR (Abcam Inc., Cambridge, MA) or ET-BR (Biodesign, Saco, ME) at 1:100 dilution for $30 \mathrm{~min}$ followed by the secondary antibody for $30 \mathrm{~min}$ and color development with DAB as provided in the DakoCytomation EnVision+ System-HRP (DakoCytomation, Carpinteria, CA). All slides were stained simultaneously to minimize variation in staining intensity. The software MetaMorph v. 6.2r4 (Universal Imaging Corporation, Downingtown, PA) interfaced with a Nikon Labophot microscope equipped with a QiCam Fast Cooled high resolution CCD camera $(1392 \times 1040$ pixels $)$ was used for image analysis. Pulmonary arteries were defined as vessels that accompanied airways (veins are interlobular). At least 20 arteries from different $400 \times$ fields were evaluated from each section. Only arteries $20-150 \mu \mathrm{m}$ in external diameter were analyzed. A threshold was defined for positive antibody staining in comparison with nonimmune serum controls. Positive pixels were expressed as a percentage of total tissue area, averaged over six random fields per sample (18).

Quantitation of collagen and elastin. Lung sections were stained with either Sirius red F3BA (Picric Acid Sirius Red stain; Rowley Biochemical Institute, Rowley, MA) or Verhoeff's elastic tissue stain for the assessment of vascular collagen and vascular elastin, respectively. For collagen quantitation, the tunica media and area around the vessel with collagen staining was selected, and the collagen staining measured using the image analysis software and expressed as a percentage of this area (vessel + perivascular area). For elastin quantitation, the vessel area including the external elastic lamina was selected, and the area occupied by elastin was measured and expressed as a percentage of the vessel area. All slides were stained simultaneously to minimize variation in staining intensity, and all measurements were done by a single observer masked to the slide identity.

Collagen in homogenized lung extracts was also measured using the Sircol soluble collagen assay (Biocolor Ltd., Newtonabbey, Northern Ireland) as described in the manufacturer's protocol. The dye reagent binds specifically to the $[\mathrm{Gly}-\mathrm{X}-\mathrm{Y}] \mathrm{n}$ helical structure found in all collagens and this assay provides an estimate of total lung collagen.
Quantitative real-time PCR analysis of ET-1, ET-AR, ET-BR, tropoelastin, collagen I, and III expression. One microliter of total RNA was reverse transcribed to cDNA using the SuperScript III First-Strand Synthesis System for RT-PCR kit from Invitrogen. The random hexamers were used as primers for cDNA synthesis. The real-time PCR was done on a Bio-Rad iCycler (Bio-Rad, Hercules, CA) and analyzed by its software. SYBR Green PCR Master Mix was used for the PCR (Applied Biosystems, Foster City, CA). The mix was optimized for real-time PCR analysis using SYBR Green 1 Dye, AmpliTaq Gold DNA Polymerase, dNTP with dUTP, Passive Reference 1, and optimized buffer components. The primers used were: ET-1: $5^{\prime}$ - CCTGGACATCATCTGGGTC- ${ }^{\prime}, 5^{\prime}$-TGTGGCCTTATTGGGAAG-3'; ET-AR: 5'-GGTGGCTCTTTGGGTTCT-3', 5'-GACGCTGTTTGAGGTGCT-3'; ET-BR: 5'-TGCGAAATGCTCAGGAAG-3'，5'-ACGAGGACCAGGCAGAAG-3'; tropoelastin: 5'-TGGTATTGGTGGCATCGG-3', 5' -CCTTGGCTTTGACTCCTGTG-3'; collagen I $\alpha$ chain 1 : $5^{\prime}$-GACGCCATCAAGGTCTACTG-3', $5^{\prime}$-GAATCCATCGGTCATGCTCT- $3^{\prime}$; collagen III $\alpha$ chain 1:5'-TTGTTGGAGTTGGAGGTGA-3'， 5'-AGTTCCGAGCAGGGTTTT3'; and 18S RNA: 5'-CTTTGGTCGCTCGCTCCT-3' and 5'-GATAAATGCACGCATCCC- $3^{\prime}$. The normalized mRNA/18S rRNA ratios were standardized to mean mRNA level of air control mice. If samples from animals of different ages were compared, the average expression seen in air-exposed animals on d 1 was used to normalize values.

Statistical analyses. Results were analyzed by two-way ANOVA (age $\times$ hypoxia and hypoxia $\times$ BQ610) followed by Student-Newman-Keuls multiple comparison test if significant differences were observed.

\section{RESULTS}

Endothelin-1 protein and mRNA expression in lung extracts. ET-1 protein concentrations and mRNA expression were higher soon after birth $(1 \mathrm{~d})$, compared with 2 wk of age (Fig. 1). Hypoxia attenuated the normal postnatal decrease in both ET-1 protein and mRNA, such that levels at 2 wk of age in hypoxia were higher than in air (Fig. 1). BQ610 increased ET-1 protein concentrations and mRNA in the hypoxiaexposed but not in the normoxic animals (Fig. 1).

ET-AR staining and mRNA expression. ET-AR staining demonstrated a trend toward a decrease with increasing age $(p=0.08)$ (Figs. 1 and 2). Hypoxia increased ET-AR staining at $1 \mathrm{~d}$ of age compared with air controls, but this difference was no longer significant by $14 \mathrm{~d}$ (Fig. 1). BQ-610 markedly increased ET-AR staining in both air and hypoxia (Fig. 2). The magnitude of qualitative difference in staining (Fig. 2) appears greater than the differences noted quantitatively (Fig. $1 \mathrm{~B})$ as the immunohistochemical staining is corrected for tissue area, which is higher soon after birth. ET-AR mRNA expression declined by $14 \mathrm{~d}$ but was not altered by hypoxia (Fig. 1). There was a trend toward reduction of ET-AR mRNA expression by BQ610 during hypoxia (Fig. 1).

ET-BR staining and mRNA expression. ET-BR staining reduced with increasing age, and this reduction was attenuated with hypoxia or BQ-610 (Figs. 1 and 3). ET-BR mRNA expression declined by $14 \mathrm{~d}$, and was also lower at $1 \mathrm{~d}$ of age while in hypoxia. ET-BR mRNA was reduced by BQ610 during hypoxia, but not during normoxia (Fig. 1).

Vascular collagen. At $1 \mathrm{~d}$ of age, marked collagen staining was noted in the interstitium as well as the tunica adventitia and tunica media, but by $14 \mathrm{~d}$, interstitial staining decreased and most collagen staining was in adventitia (Fig. 4). The vascular collagen volume immediately after birth was $39 \pm$ $2 \%$. By $14 \mathrm{~d}$, vascular collagen had decreased by two-thirds in air controls, and to a lesser extent in hypoxia-exposed animals, so that there was a moderate increase (20\%) in vascular collagen volume with hypoxia (13.4 $\pm 0.7 \%$ of vascular area 
A
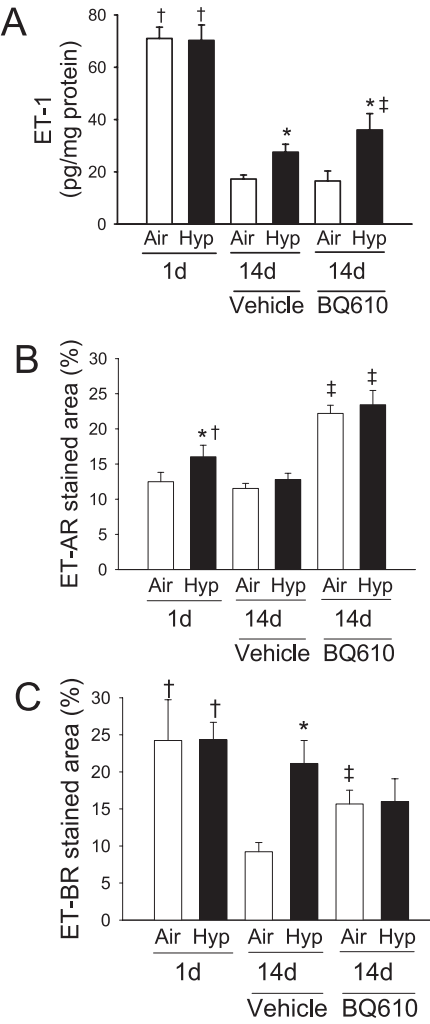

Figure 1. Effects of 1 and 14 d hypoxic exposure $\left(12 \% \mathrm{O}_{2}, 1 \mathrm{~atm}\right)$ on $(A)$ ET-1 protein and steady-state mRNA expression, $(B)$ ET-AR protein and steady-state mRNA expression, and $(C)$ ET-BR protein and steady-state mRNA expression in neonatal mouse lung. Animals exposed to air or hypoxia for $14 \mathrm{~d}$ were administered either vehicle or BQ-610 (ET-AR antagonist) daily from birth. Results are means $\pm \mathrm{SE}, n=6-8$ mice/group. ${ }^{*} p<0.05 v s$ respective air control groups; $\dagger p<0.05$ vs respective 14-d groups; $\ddagger p<0.05$ $v s$ respective vehicle controls.

in air versus $16.0 \pm 0.8 \%$ in hypoxia; $p<0.05$ ). ET-AR blockade reduced collagen volume to the same extent regardless of $\mathrm{O}_{2}$ level. In mice administered BQ610 versus vehicle, vascular collagen declined from $13.4 \pm 0.7 \%$ to $8.8 \pm 0.5 \%$ $(p<0.05)$ in air animals, and from $16.0 \pm 0.8 \%$ to $8.1 \pm 1 \%$ $(p<0.05)$ in the hypoxia group. A similar pattern of response was noted when vessels of different sizes (20-50 $\mu \mathrm{m}, 50-100$ $\mu \mathrm{m}$, and $100-150 \mu \mathrm{m}$ ) were evaluated individually (data not shown), although smaller vessels had less adventitial collagen. A similar attenuation of the postnatal decrease with hypoxia, and decreases with BQ610 were seen when whole lung collagen was analyzed by the Sircol method, although the change in collagen with BQ610 was not statistically significant (Fig. 4).

No significant changes were noted in collagen I and III expression with either hypoxia or BQ610 (Table 1), although there was a nonsignificant trend for reduction in collagen III with both hypoxia and BQ610.

Vascular elastin. Elastin staining was most intense in the internal and external elastic laminae (Fig. 5). Vascular elastin volume immediately after birth was $22.9 \pm 1 \%$, and decreased by two-thirds in air controls, and to a lesser degree in hypoxiaexposed animals, such that hypoxia-exposed animals had 60\% more elastin at $14 \mathrm{~d}$ compared with air-exposed animals
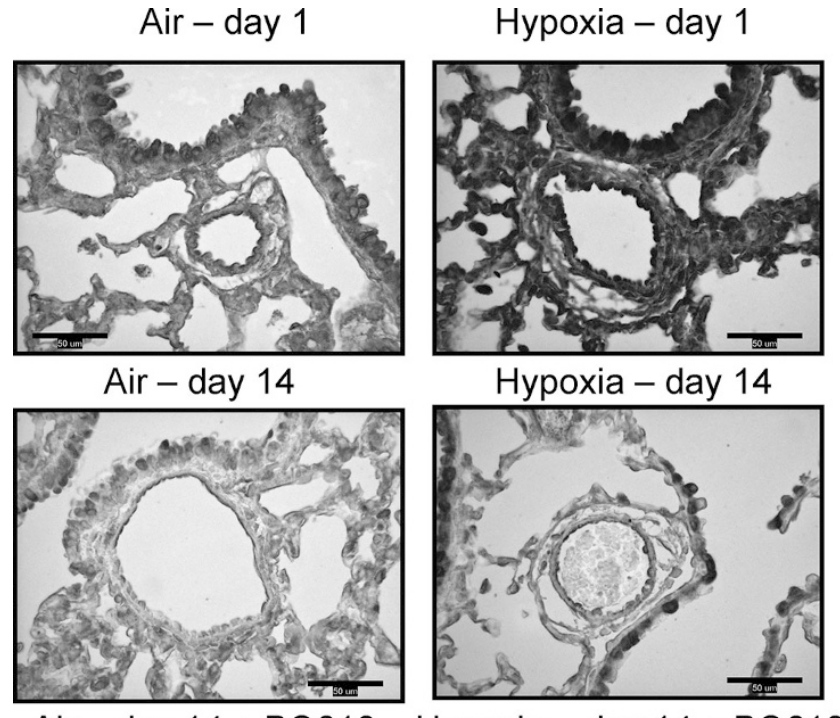

Hypoxia - day 14
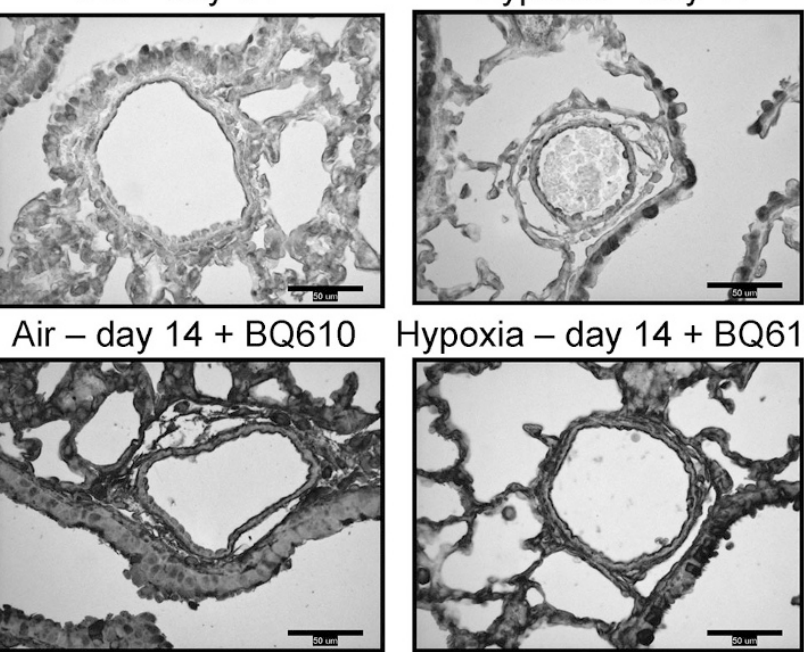

Hypoxia - day $14+$ BQ610

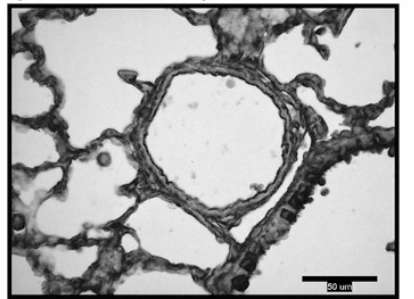

Figure 2. Effects of hypoxia and/or BQ610 on immunohistochemical staining for ET-AR in neonatal mouse lungs after 1 and $14 \mathrm{~d}$ of hypoxic exposure. $(400 \times$; calibration bar $=50 \mu \mathrm{m}$.)

(7.6 $\pm 0.5 \%$ of vascular area in air versus $12.2 \pm 0.5 \%$ in hypoxia, $p<0.05$ ). ET-AR blockade did not reduce elastin staining (hypoxia-vehicle: $12.2 \pm 0.5 \%$ versus hypoxiaBQ610: $14.2 \pm 0.8 \%$; NS). A similar pattern of response was noted when vessels of different sizes were evaluated individually (data not shown), although smaller vessels had less vascular elastin compared with larger vessels. Similar changes were seen in vascular elastin when absolute elastin volumes, rather than elastin volumes normalized to vessel area, were analyzed (data not shown). A corresponding increase in tropoelastin mRNA expression was noted with hypoxia (Table 1). BQ610 inhibited this hypoxia-induced increase in tropoelastin expression, but did not affect elastin staining (Table 1).

\section{DISCUSSION}

The major findings of the current study are a) in the neonatal mouse lung, ET-1 concentrations and mRNA expression normally decline postnatally, and hypoxia attenuates this process; and b) vascular collagen fiber volume normally decreases after birth, and this normal reduction is impaired by hypoxia in an ET-AR-dependent fashion, without altering steady-state collagen I or III mRNA levels. These observations, coupled with the finding that ET-AR blockade also reduces vascular collagen content in normoxia-exposed mice, suggests that ET-1 is necessary for collagen maintenance and deposition in the pulmonary vasculature during the neonatal period. The finding that collagen deposition is altered in the absence of changes in mRNA expression suggests that hyp- 


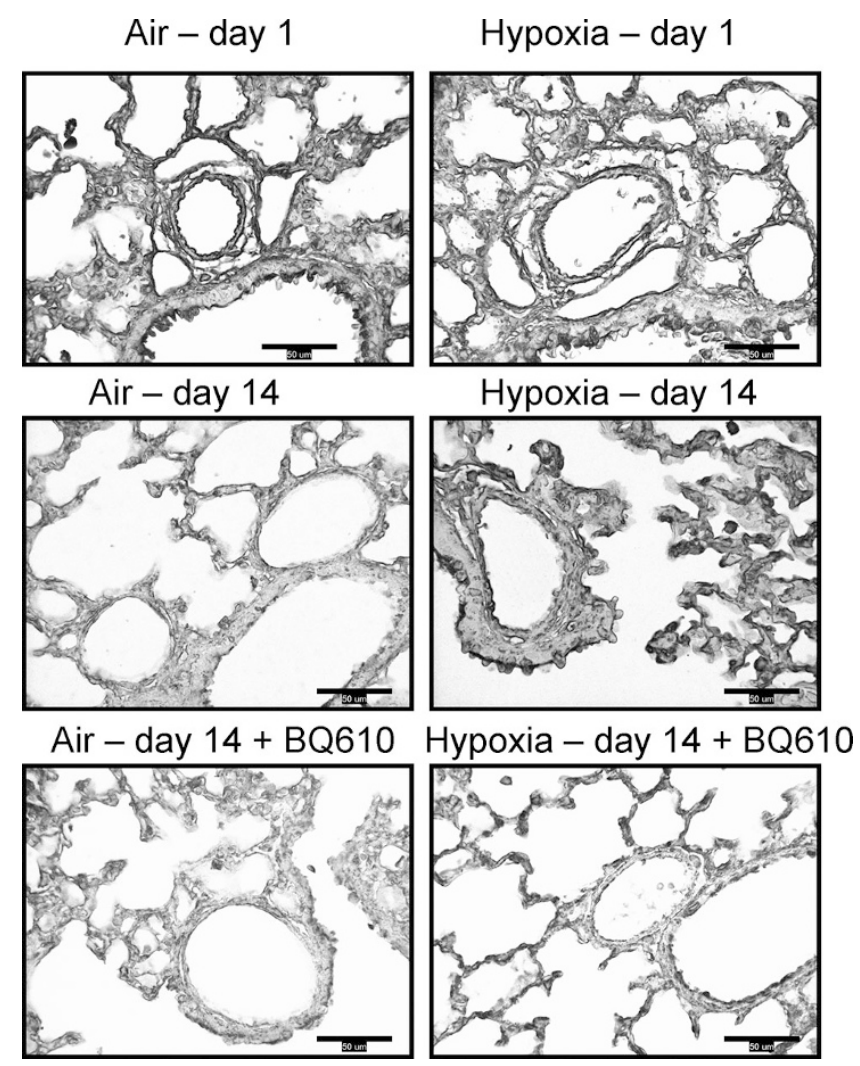

Figure 3. Effects of hypoxia and/or BQ610 on immunohistochemical staining for ET-BR in neonatal mouse lungs after 1 and $14 \mathrm{~d}$ of hypoxic exposure. ( $400 \times$; calibration bar $=50 \mu \mathrm{m}$.)

oxia and ET-1 may reduce normal postnatal collagen breakdown/turnover in the newborn mouse lung. Pulmonary vascular elastin also declined postnatally, and this normal decrease was again impaired by hypoxia. In contrast to collagen, hypoxia-induced increases in vascular elastin content were not modulated by ET-AR blockade, although ET-AR blockade was highly effective in inhibiting steady-state tropoelastin mRNA levels. As in the case of collagen, these findings suggest that hypoxia-induced alterations in pulmonary vascular elastin content are dependent on posttranslational alterations in elastin deposition or turnover. We have previously shown that ET-AR blockade prevents and partially reverses hypoxia-induced pulmonary vascular remodeling in newborn mice (8), and the current study demonstrates that ET-AR blockade may selectively increase collagen but not elastin turnover in the newborn lung.

The postnatal decrease in ET-1 protein concentrations and in ET-1, ET-AR, and ET-BR mRNA expression that we observed is consistent with similar decreases seen in piglets by Levy et al. (19), and extends work done by Adur et al. (20), who showed that ET-AR and ET-BR are maximal in the first or second day after birth in mice. These results are to be expected, as transition from the relatively hypoxic in utero environment to the better oxygenated postnatal environment should reduce ET-1 and its signaling, which is normally up-regulated by hypoxia. The normal postnatal reduction in ET-1 and ET-AR is therefore favorable for, and may contribute toward, the normal physiologic changes in pulmonary

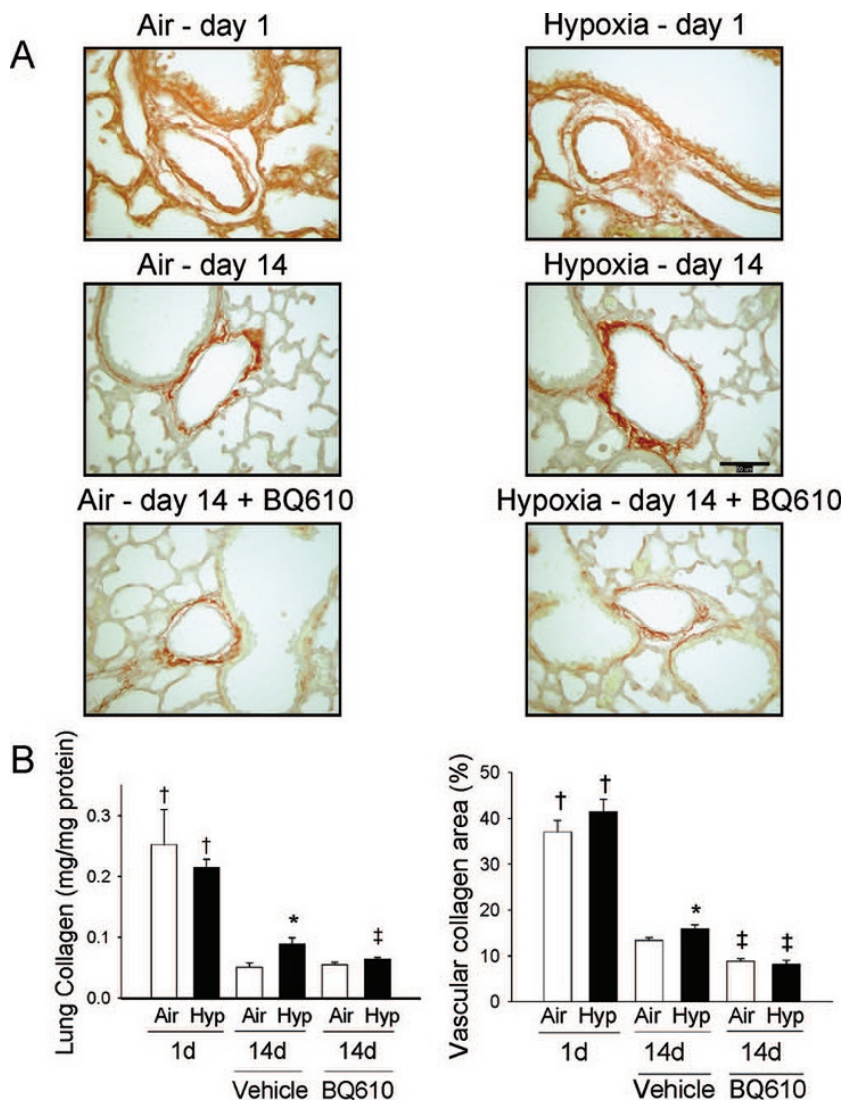

Figure 4. Effects of hypoxia and/or BQ610 on perivascular collagen staining in neonatal mouse lung after 1 and $14 \mathrm{~d}$ of hypoxic exposure. Lung sections were stained with picrosirius red (PASR) for collagen. (A) Representative photomicrographs of pulmonary arteries $(400 \times$; calibration bar $=50 \mu \mathrm{m})$. (B) Total lung collagen by Sircol method, and vascular collagen area by quantitative image analysis of PASR stained sections. Results are means \pm SE, $n=120-160$ pulmonary arteries from 6 to 8 mice/group. ${ }^{*} p<0.05$ vs respective air control groups, $\dagger p<0.05 v s$ respective 14-d groups, $\ddagger p<0.05$ $v s$ respective vehicle controls.

blood flow (decrease in vascular resistance, increase in flow) and anatomical changes (reduction in vascular wall thickness and extracellular matrix) that occur with transition from the in utero environment to postnatal life. Consistent with this explanation, we observed that hypoxia inhibited the normal postnatal decrease in ET-1 protein and mRNA expression. However, although hypoxia inhibited the normal decrease in ET-BR staining at $14 \mathrm{~d}$, no decrease in ET-AR staining or in ET-AR or ET-BR mRNA was observed. As the increase in ET-BR staining occurred without a change in ET-BR mRNA, posttranslational changes in receptor number or density may be involved. BQ610, only under hypoxic conditions, showed a trend toward reduction of ET-1 and ET-AR mRNA, and reduced ET-BR mRNA expression. We speculate that when ET-AR is blocked, increased levels of ET-1 acting unopposed via ET-BR may possibly reduce expression of ET-1 and its receptors. The dissociation between increased ET-AR staining seen in BQ610-exposed animals (both in normoxia and hypoxia) and the lack of change or decrease in ET-AR mRNA may indicate a reduction in ET-AR turnover due to receptor binding by the antagonist. In this setting, the increase in ET-AR staining represents an increase in "blocked" receptors and not increased ET-AR activity. 
Table 1. Results of quantitative real-time RT-PCR for tropoelastin, collagen I, and collagen III from newborn mouse lung following either hypoxia $\left(12 \% \mathrm{O}_{2}\right)$ or air from birth to 2 wk of age, while receiving either vehicle or BQ610

\begin{tabular}{ccccc}
\hline \multicolumn{1}{c}{ Genes } & Air + vehicle & Air + BQ610 & Hypoxia + vehicle & Hypoxia + BQ610 \\
\hline Collagen I & $1.00 \pm 0.09$ & $1.18 \pm 0.17$ & $1.01 \pm 0.11$ & $0.87 \pm 0.12$ \\
Collagen III & $1.00 \pm 0.28$ & $0.72 \pm 0.25$ & $0.52 \pm 0.16$ & $0.26 \pm 0.05$ \\
Tropoelastin & $1.00 \pm 0.40$ & $0.42 \pm 0.17$ & $3.98 \pm 0.53^{*}$ & $0.45 \pm 0.10^{* *}$ \\
\hline
\end{tabular}

By two-way ANOVA, hypoxia increased while BQ610 decreased tropoelastin expression $(p<0.05)$. There was a nonsignificant trend for reduction in collagen III with both hypoxia and BQ610 $(p=0.1)$. There was a significant interaction between hypoxia and BQ610 for tropoelastin $(p<0.01)$ but not for collagen I or III ( $p=0.22$ for collagen I, $p=0.71$ for collagen III). $(n=6-8 / \mathrm{gp}$; mean $\pm \mathrm{SE}, * p<0.05 v s$ respective air control, $* * p<0.05 v s$ respective vehicle groups).

Vascular collagen fiber volume decreased over the first two postnatal weeks, and hypoxia attenuated this decrease. As hypoxia did not significantly change collagen mRNA expression, an impairment of collagen turnover/breakdown with hypoxia may be responsible for the relative accumulation of collagen. Collagen turnover is primarily regulated by matrix metalloproteinases (MMP). We have recently demonstrated

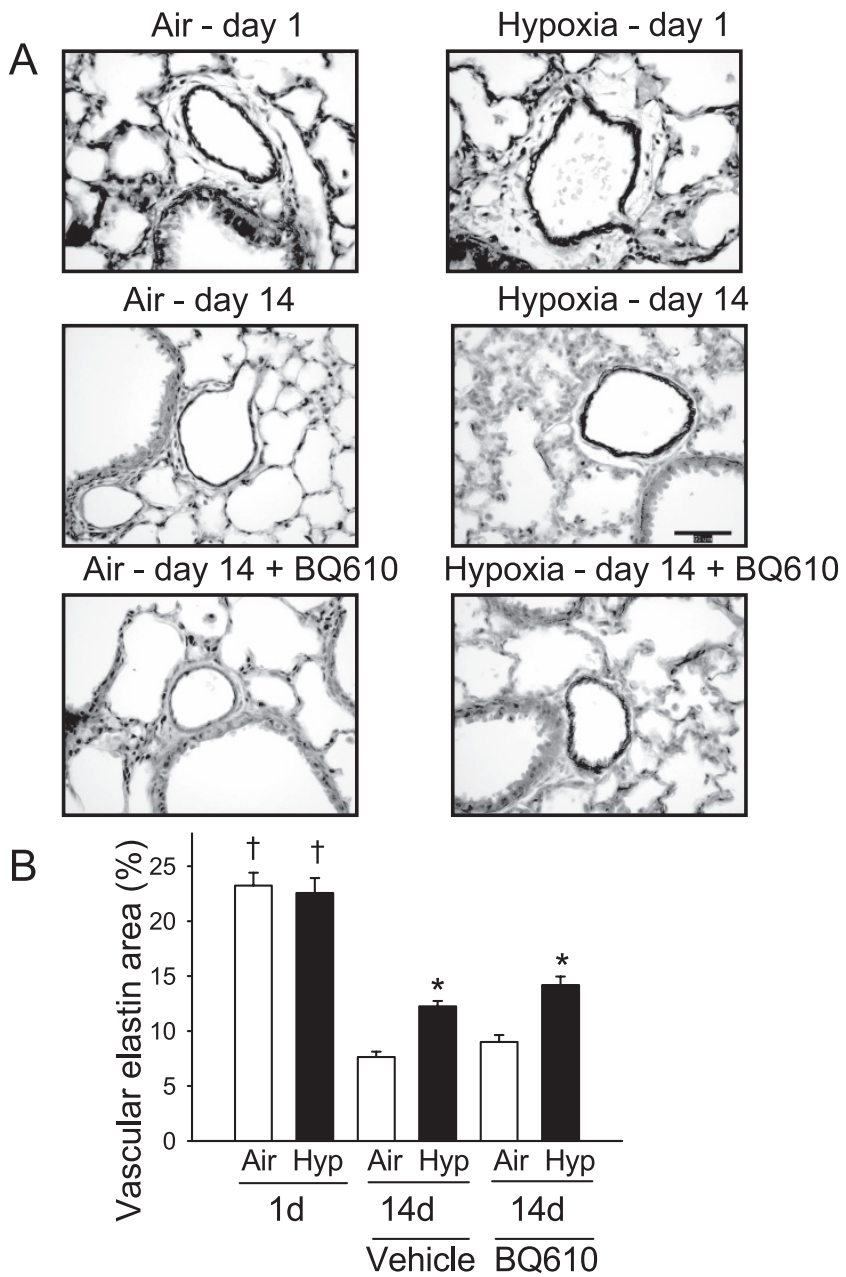

Figure 5. Effects of hypoxia and/or BQ610 on vascular elastin staining and elastin mRNA expression on pulmonary arteries in neonatal mouse lung after 1 and $14 \mathrm{~d}$ of hypoxic exposure. Lung sections were stained with Verhoeff's elastic stain for elastin. (A) Representative photomicrographs of pulmonary arteries $(400 \times$; calibration bar $=50 \mu \mathrm{m}) .(B)$ Vascular elastin area in pulmonary arteries was measured by quantitative image analysis in elastinstained lung sections. Results are means $\pm \mathrm{SE}, n=120-160$ pulmonary arteries from 6 to 8 mice/group. ${ }^{*} p<0.05$ vs respective air control groups, $\dagger p<$ 0.05 vs respective 14-d groups, $\ddagger p<0.05$ vs respective vehicle controls. that hypoxia reduces MMP-2, the major gelatinase in the newborn lung, and that MMP-2 deficient (MMP-2 null) mice have abnormal arterial remodeling and collagen accumulation (Ambalavanan et al. Pediatric Academic Societies' meeting, May 14-17, 2005, Washington DC). The hypoxia-induced persistence of vascular collagen may contribute to pulmonary hypertension. In chronic hypoxia-exposed adult rats, the antifibrotic agents beta-aminopropionitrile or cis-4-hydroxy-Lproline prevent pulmonary hypertension, right ventricular hypertrophy, and vascular collagen accumulation (21,22). BQ610 decreased perivascular collagen content under both normoxic and hypoxic conditions, although it did not change collagen expression significantly. This suggests that ET-AR blockade increases collagen breakdown, and therefore indicates that ET-1 may decrease collagen breakdown and increase collagen content by mechanisms that need to be identified in this model. These results are consistent with those of other investigators who have demonstrated that ET-AR blockade regresses vascular fibrosis in other organs (23). We are currently investigating the mechanisms by which ET-1 may interact with MMP, and postulate that the decrease in collagen with ET-AR blockade may contribute to a reduction in HPVR.

In our newborn mouse model, hypoxia increased elastin in pulmonary arteries. Similar increases in vascular elastin with hypoxia have been seen in newborn rats (24) and calves (25). Our model showed an increase in vascular elastin staining and tropoelastin expression with hypoxia, but BQ610 did not reduce elastin although it reduced tropoelastin mRNA. These results suggest that whereas hypoxia increases tropoelastin and thereby elastin, ET-AR antagonists do not reduce elastin despite reducing mRNA expression, probably due to an impairment of elastin breakdown with hypoxia. A similar decrease in elastin breakdown with hypoxia has been seen in newborn piglets wherein hypoxia had no effect on tropoelastin mRNA but led to increased tropoelastin protein deposition (26).

Other fetal (27) and neonatal models (7) have shown that ET-AR antagonists prevent pulmonary vascular remodeling, but changes in extracellular matrix (ECM) have not been the focus of these studies. The ECM regulates cellular migration, proliferation, and differentiation, and is involved in organizing airway branching, alveolar septal development (28), and the postnatal plasticity of the pulmonary circulation $(25,29)$. HPVR may result from impairment of this regulation $(25,29)$. It is likely that other ECM molecules, in addition to collagen and elastin, also play a role in regulating the effects of hypoxia on the vasculature. 
The advantage of our model is that lung development of mice in the first two postnatal weeks is similar to that in humans from the 24th week of gestation through the first $2 \mathrm{y}$ of age (30). In addition, transgenic (either "knockout" or "overexpression") mice can be used for evaluating effects of specific genes or their absence on lung alveolar and vascular development. There are limitations to the newborn mouse model, as in all animal models. This model is not directly extrapolatable to human neonates due to interspecies differences. Hemodynamic measurements in newborn mice are also difficult. Models of chronic alveolar hypoxia, although reproducible, do not closely simulate the pathophysiology of pulmonary hypertension in human neonates. Also, due to agespecific effects, fetal hypoxia, hypoxia soon after birth, and hypoxia starting a week after birth have different physiologic and structural effects. A limitation of our real-time RT-PCR analysis is that whole-lung homogenates from one point in time were used, and hence these results represent changes in gene expression of the lung parenchyma and airways, as well as the vasculature at that point in time. However, these results generate hypotheses that can be tested by more precise techniques such as laser capture microdissection and in situ hybridization. Chronic hypoxic exposure in the developing animal (unlike the adult) leads to impairment of lung alveolar and vascular development $(24,31,32)$, in addition to growth retardation. This hypoxia-induced impairment of alveolar and vascular development in lung may contribute to pulmonary hypertension, in addition to HPVR.

In summary, chronic hypoxia exposure in neonatal mice attenuates the normal postnatal decrease in ET-1 and its receptors, and leads to vascular collagen and elastin accumulation that may be important in vascular remodeling. Increased collagen seems to be mediated mainly by a decrease in breakdown/turnover whereas increased elastin is mediated both by increased expression and decreased breakdown. ET-AR blockade reduces perivascular collagen but not vascular elastin content. Many mechanisms involving mediators such as nitric oxide, prostacyclin, reactive oxygen species, and heat shock proteins, in addition to ET-1, are involved in HPVR. It may therefore be expected that ET-AR blockade would reverse only some of the effects of chronic hypoxia. Knowledge of the mechanisms that are modulated and those that are not modulated by ET-AR antagonists provides useful information for developing therapeutic strategies that may best be combined with these endothelin antagonists, to achieve complementary beneficial effects.

\section{REFERENCES}

1. Murphy JD, Vawter GF, Reid LM 1984 Pulmonary vascular disease in fatal meconium aspiration. J Pediatr 104:758-762

2. Walsh-Sukys MC 1993 Persistent pulmonary hypertension of the newborn. The black box revisited. Clin Perinatol 20:127-143

3. Goodman G, Perkin RM, Anas NG, Sperling DR, Hicks DA, Rowen M 1988 Pulmonary hypertension in infants with bronchopulmonary dysplasia. J Pediatr 112:67-71
4. Steinhorn RH, Fineman JR 1999 The pathophysiology of pulmonary hypertension in congenital heart disease. Artif Organs 23:970-974

5. Rabinovitch M, Gamble WJ, Miettinen OS, Reid L 1981 Age and sex influence on pulmonary hypertension of chronic hypoxia and on recovery. Am J Physiol 240:H62-H72

6. Stenmark KR, Fasules J, Hyde DM, Voelkel NF, Henson J, Tucker A, Wilson H, Reeves JT 1987 Severe pulmonary hypertension and arterial adventitial changes in newborn calves at 4,300 m. J Appl Physiol 62:821-830

7. Perreault T, Berkenbosch JW, Barrington KJ, Decker ER, Wu C, Brock TA, Baribeau J 2001 TBC3711, an ET(A) receptor antagonist, reduces neonatal hypoxiainduced pulmonary hypertension in piglets. Pediatr Res 50:374-383

8. Ambalavanan N, Bulger A, Murphy-Ullrich J, Oparil S, Chen YF 2005 Endothelin-A receptor blockade prevents and partially reverses neonatal hypoxic pulmonary vascular remodeling. Pediatr Res 57:631-636

9. Kelly DA, Hislop AA, Hall SM, Haworth SG 2002 Correlation of pulmonary arterial smooth muscle structure and reactivity during adaptation to extrauterine life. J Vasc Res 39:30-40

10. Ziegler JW, Ivy DD, Kinsella JP, Abman SH 1995 The role of nitric oxide, endothelin, and prostaglandins in the transition of the pulmonary circulation. Clin Perinatol 22:387-402

11. Ortega Mateo A, de Artinano AA 1997 Highlights on endothelins: a review. Pharmacol Res 36:339-351

12. Wong J, Vanderford PA, Fineman JR, Chang R, Soifer SJ 1993 Endothelin-1 produces pulmonary vasodilation in the intact newborn lamb. Am J Physiol 265:H1318-H1325

13. Bradley LM, Czaga JF, Goldstein RE 1990 Circulatory effects of endothelin-1 in newborn piglets. Am J Physiol 259:H1613-H1617

14. Barnard JW, Barman SA, Adkins WK, Longnecker GL, Taylor AE 1991 Sustained effects of endothelin-1 on rabbit, dog, and rat pulmonary circulation. Am J Physiol 261:H479-H486

15. Chen YF, Oparil S 2000 Endothelial dysfunction in the pulmonary vascular bed Am J Med Sci 320:223-232

16. Ambalavanan N, Philips JB 3rd, Bulger A, Oparil S, Chen YF 2002 Endothelin-A receptor blockade in porcine pulmonary hypertension. Pediatr Res 52:913-921

17. DiCarlo VS, Chen SJ, Meng QC, Durand J, Yano M, Chen YF, Oparil S 1995 ET-A Receptor antagonist prevents and reverses chronic hypoxia-induced pulmonary hypertension in rat. Am J Physiol 269:L690-L697

18. Brey EM, Lalani Z, Johnston C, Wong M, McIntire LV, Duke PJ, Patrick CW Jr 2003 Automated selection of DAB-labeled tissue for immunohistochemical quantification. J Histochem Cytochem 51:575-584

19. Levy M, Souil E, Sabry S, Favatier F, Vaugelade P, Mercier JC, Dall'Ava-Santucci J, Dinh-Xuan AT 2000 Maturational changes of endothelial vasoactive factors and pulmonary vascular tone at birth. Eur Respir J 15:158-165

20. Adur J, Takizawa S, Quan J, Uchide T, Saida K 2003 Increased gene expression and production of murine endothelin receptors after birth. Biochem Biophys Res Commun 305:700-706

21. Kerr JS, Riley DJ, Frank MM, Trelstad RL, Frankel HM 1984 Reduction of chronic hypoxic pulmonary hypertension in the rat by beta-aminopropionitrile. J Appl Physiol 57:1760-1766

22. Poiani GJ, Tozzi CA, Choe JK, Yohn SE, Riley DJ 1990 An antifibrotic agent reduces blood pressure in established pulmonary hypertension in the rat. J Appl Physiol 68:1542-1547

23. Boffa JJ, Tharaux PL, Dussaule JC, Chatziantoniou C 2001 Regression of renal vascular fibrosis by endothelin receptor antagonism. Hypertension 37:490-496

24. Meyrick B, Reid L 1982 Normal postnatal development of the media of the rat hilar pulmonary artery and its remodeling by chronic hypoxia. Lab Invest 46:505-514

25. Durmowicz AG, Parks WC, Hyde DM, Mecham RP, Stenmark KR 1994 Persistence, re-expression, and induction of pulmonary arterial fibronectin, tropoelastin, and type I procollagen mRNA expression in neonatal hypoxic pulmonary hypertension. Am J Pathol 145:1411-1420

26. Kitley RM, Hislop AA, Hall SM, Freemont AJ, Haworth SG 2000 Birth associated changes in pulmonary arterial connective tissue gene expression in the normal and hypertensive lung. Cardiovasc Res 46:332-345

27. Ivy DD, Parker TA, Zeigler JW, Galan HL, Kinsella JP, Tuder RM, Abman SH 1997 Prolonged endothelin A receptor blockade attenuates chronic pulmonary hypertension in the ovine fetus. J Clin Invest 99:1179-1186

28. McGowan SE 1992 Extracellular matrix and the regulation of lung development and repair. FASEB J 6:2895-2904

29. Rabinovitch M 1996 Cell-extracellular matrix interactions in the ductus arteriosus and perinatal pulmonary circulation. Semin Perinatol 20:531-541

30. Burri P 1997 Structural aspects of prenatal and postnatal development and growth for the lung. In: McDonald JA (ed) Lung Growth and Development. Marcel Dekker Inc., New York, pp 1-35.

31. Vicencio AG, Eickelberg O, Stankewich MC, Kashgarian M, Haddad GG 2002 Regulation of TGF-beta ligand and receptor expression in neonatal rat lungs exposed to chronic hypoxia. J Appl Physiol 93:1123-1130

32. Sekhon HS, Thurlbeck WM 1996 Lung morphometric changes after exposure to hypobaria and/or hypoxia and undernutrition. Respir Physiol 106:99-107 